\title{
Water Flooding and Viscous Fingering in Fracture and Porous Media by Lattice Boltzmann Method
}

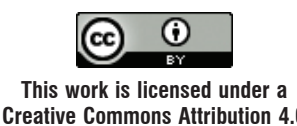

Creative Commons Attribution 4.0 International License

\author{
Y. Shiri, ${ }^{a}$ H. Hassani, ${ }^{b},{ }^{, *}$ M. Nazari, ${ }^{c}$ and M. Sharifi ${ }^{a}$ \\ ${ }^{a}$ Department of Petroleum Engineering, Amirkabir University of \\ Technology (Polytechnic of Tehran), Tehran, P.O. Box: 15875-4413, Iran \\ of Technology (Polytechnic of Tehran), Tehran, \\ P.O. Box: 15916-34311, Iran \\ 'Faculty of Mechanical Engineering, Shahrood University \\ of Technology, Shahrood, P.O. Box: 36199-95161, Iran
}

${ }^{b}$ Department of Mining and Metallurgy Engineering, Amirkabir University

doi: 10.15255/CABEQ.2017.1203

Original scientific paper

Received: August 25, 2017

Accepted: February 21, 2018

\begin{abstract}
The study of fluid front in porous media in enhanced oil recovery is important. The purpose of this study is to simulate water flooding, and investigate the factors affecting the fluid front across a microfracture and simple porous media using Shan-Chen type of the Lattice Boltzmann Method (LBM). Various factors, including velocity and dynamic viscosity that define the capillary number and wettability are considered. Independently, the increase in velocity and dynamic viscosity ratio results in viscous fingering and its narrowness. Increasing the wettability of the displacing fluid decreases viscous fingering, and as a result, it makes the fluid move in piston form. The lowest sweep efficiency occurs when the displacing fluid has a neutral wettability. Simulation results show the strength and accuracy of Shan-Chen type of LBM in fluid front tracking in porous media in pore scale.
\end{abstract}

Keywords:

fluid front in porous media, Lattice Boltzmann method, viscous fingering

\section{Introduction}

Fluid flow in porous media has a very important role in various industries, such as resin transfer modeling, filter analysis, groundwater transfer, biomechanics, and oil recovery. Replacing a fluid with another fluid for the preferential flow path of injecting fluid, which creates its own unique pattern of fluid flow according to the environmental and fluid characteristics, is one of the challenging issues in industries, especially in oil resource recovery. For instance, tongues of water are of limiting parameters in EOR. Fluid front and viscous fingering in immiscible oil displacement are very important. When a less viscous fluid pushes a fluid with a higher viscosity, the interface between the two fluids becomes unstable and the thickness of the fluid front oscillates. As a results, it is not always consistent with its estimated value ${ }^{1-3}$. Disturbances occur at the interface of the two fluids in both the laboratory and the numerical study ${ }^{2-4}$. In addition, the stability of the fluid front, because of the continuous development of the flow across the unstable state that causes droplets, is very important. The viscosity of two fluids, surface tension, wettability, the rel-

"Corresponding author, Email: hhassani@aut.ac.ir,

Tel: +989121758210, Fax: +982166405846 ative velocity of fluids, porous media structure and geometry of grain are the determining factors in viscous fingering and fluid front shape ${ }^{5,6}$.

Cinar et al. have investigated the two-phase flow regime and the effect of gravity, viscosity, capillary force and surface tension of two fluids in two parallel porous layers by finite difference and streamline methods, and they also experimented it on glass beads ${ }^{7}$. Maaref et al. have investigated the effect of wettability, heterogeneity, and viscosity differences in the process of water and oil displacement in glass micro-model and its numerical solution with finite element method. The results showed a strong correlation between the numerical method and laboratory observation, and the increase in EOR caused by higher viscosity and wettability of the injecting fluid as well as the decrease in heterogeneity in porous media ${ }^{8}$.

In the absence of any analytical solution for the fluid flow, the numerical simulation will work. The most noticeable method is discretizing of equation by using finite difference and finite element methods on the macroscopic scale as well as the useful Lattice Boltzmann method (LBM) in the mesoscopic scale ${ }^{9}$. Different Computational Fluid Dynamic (CFD) techniques including Volume of the Void (VOF) and the Level-set method are used to simu- 
late two-component or two-phase fluids, but their interface can only be assessed in large-scale, and the information is usually ignored in small scales. LBM has recently become one of the most useful methods for calculating fluid dynamics. The convenience of using a variety of boundary conditions along with intrinsic network has valued LBM for simulating flow and heat transfer in complex systems with special geometrical dimensions ${ }^{10-13}$. Some advantages of LBM are its ability to evaluate the factors involved in viscous fingering in the mesoscopic scale, reduce computational time, perform parallel computations, be implemented easily, and continuously track the fluid front within the time rather than other macroscopic and microscopic methods ${ }^{14}$. The mesoscopic nature of LBM coupled with the easy use of the boundary conditions on the solid wall has made it a powerful tool for simulating fluid flow in porous media in small scales. Also, this method has the ability to simulate fluids both in microscopic and macroscopic scales ${ }^{15}$. Different models of LBM, such as Color Gradient (Rothman-Keller) Method, Pseudo-Potential (Shan-Chen) Model, Free Energy Model, and Mean-Field Model have been developed for two-phase fluid simulation $^{16-19}$. In the Color Gradient Model, a local gradient in the phase field is used to separate the phases, and the advantages of it are the independent use of viscosity ratio, contact angle, surface tension, and density ratio ${ }^{14,20}$. Pseudo-Potential (Shan-Chen) Model uses pseudopotential coefficient for particle interaction, and its advantages are simplicity and computational efficiency. In this method, surface tension, density, and viscosity ratios are not independent ${ }^{21}$. In the Free Energy Model, the phases' interaction directly defined by the general equilibrium distribution function, which includes the pressure tensor in the collision process. In the Mean-Field Model, the appropriate force is extracted from fluid phase behavior. LBM is one of the hottest topics in recent years and Shan-Chen Model is the main body of simulation in these studies ${ }^{22}$.

At the time of injecting a low viscous fluid into a higher viscous fluid, due to the applied force with the instability in the interface of two fluids, finger-like patterns form, and its shape depends on several factors. Tracking and investigating effective factors of fluid front in viscous fingering in a microfracture is the most common model due to the existence of their analytical solutions. Various studies in the Hele-Shaw microchannel, which represent the smallest component in complex geometries, have been performed experimentally ${ }^{6,23-25}$ and numerically using $\mathrm{LBM}^{26-30}$. Studies have shown that the fingertip is narrowed by increasing the capillary number and the viscosity ratio, as wells by decreasing the wettability of the injected fluid. It is promis- ing that the Shan-Chen Model of LBM has the ability to study the fluid front ${ }^{31}$. In addition, the study of the fluid front in porous media is also important. In recent years, remarkable studies have been carried out in this regard and some parameters including the viscosity ratio, velocity, surface tension, and adhesive force have been investigated ${ }^{32-38}$. The purpose of the present study is to use Shan-Chen Model of LBM and investigate its ability to accurately examine the main factors affecting the fluid front in a microfracture and a simple porous media.

\section{Lattice Boltzmann method}

\section{Lattice Boltzmann equation}

LBM can be simplified by the Lattice Gas Automata (LGA) or by the finite difference solution of the Boltzmann equation. Both LBM and LGA can be obtained from the Navier-Stokes Equations on the macroscopic scale. For the first time in 1964, LGA was used to simulate fluid flow ${ }^{39}$. However, this method did not yield good results due to its statistical noise and its dependence on velocity. Later in 1988, LBM was introduced for simulating the fluid flow, which apart from the advantages of LGA, was numerically more efficient and more appropriate to simulate flow with high Reynolds number and more applicable in glacier flow ${ }^{40,41}$. Then, LBM was obtained by discretizing and solving the Boltzmann equation ${ }^{42}$.

\section{Shan-Chen model of LBM}

Shan-Chen model of LBM is used to simulate fluid flow with several phases and components, and is known as the Pseudo-Potential model that is based on the concept of the intermolecular interaction of fluid particles. Shan-Chen model of LBM for each phase is in equation (1), and is updated after initialization in a cycle consisting of collision, streaming, applying boundary conditions, and determining fluid variable until the stop criterion is achieved.

$$
\begin{aligned}
& f_{i}^{\sigma}\left(x+c_{i}, t+1\right)-f_{i}^{\sigma}(x, t)= \\
& =-\frac{1}{\tau_{\sigma}}\left[f_{i}^{\sigma}(x, t)-f_{i}^{\sigma(e q)}(x, t)\right]
\end{aligned}
$$

The density, velocity, and distribution function of each phase can be determined by:

$$
\begin{gathered}
\rho_{\sigma}=\sum_{i} f_{i}^{\sigma}, \quad u_{\sigma}=\frac{1}{\rho_{\sigma}} \sum_{i} f_{i}^{\sigma} c_{i} \\
f_{i}^{\sigma(e q)}=w_{i} \rho_{\sigma}\left[1+\frac{c_{i} \cdot u_{\sigma}^{e q}}{c_{s}^{2}}+\frac{\left(c_{i} \cdot u_{\sigma}^{e q}\right)^{2}}{2 c_{s}^{4}}-\frac{u_{\sigma}^{e q} \cdot u_{\sigma}^{e q}}{2 c_{s}^{2}}\right]
\end{gathered}
$$


In this method, it is assumed that the total force to one of the phases which consists of the effective nearest neighbor, gravity, and adhesion forces $\left(F^{\sigma}=F_{f}^{\sigma}+F_{s}^{\sigma}+F_{g}^{\sigma}\right)$ are as follows ${ }^{43}$.

$$
\begin{gathered}
F_{f}^{\sigma}(x)=-\psi_{\sigma}(x) G_{\sigma \bar{\sigma}} \sum_{X^{\prime}} \sum_{\bar{\sigma}=1}^{S} w_{i} \psi_{\bar{\sigma}}\left(x^{\prime}\right) c_{i} \\
F_{s}^{\sigma}(x)=-\rho_{\sigma}(x) \sum_{i} w_{i} G_{s}^{\sigma} S\left(x^{\prime}+c_{i}\right) c_{i} \\
F_{g}^{\sigma}(x)=\rho_{\sigma} g
\end{gathered}
$$

In Equation (4) $\sigma$ and $\bar{\sigma}$ are different fluid phases. The amount of $G_{\sigma \bar{\sigma}}$ represents the fluid-fluid particle interaction or the amount of attraction or repulsive force between fluid particles. The zero value indicates the miscibility of the phases, and its increase causes significant reduction in their miscibility. $G_{s}^{\sigma}$ represents fluid-solid interaction and determines the wettability. The effective density, which is introduced by Shan and Chen is as follows ${ }^{17}$.

$$
\psi_{\sigma}(x, t)=\rho_{0}\left[1-\exp \left(-\frac{\rho(x, t)}{\rho_{0}}\right)\right]
$$

For densities much smaller than 1 , this function is equal to the density $(\rho<<1 \Rightarrow \psi(\rho)=\rho)$. The total force can be used as follows:

$$
u_{\sigma}^{e q}=u^{\prime}+\frac{\tau_{\sigma} F_{\sigma}}{\rho_{\sigma}}
$$

In this equation, $u^{\prime}$ is the velocity of all phases and is defined as follows:

$$
u^{\prime}=\frac{\sum_{\sigma} \frac{1}{\tau_{\sigma}} \sum_{i} f_{i}^{\sigma} c_{i}}{\sum_{\sigma} \frac{1}{\tau_{c}} \rho_{\sigma}}
$$

By considering intermolecular force, fluid behavior will be non-ideal, and the equation of state is as follows:

$$
P=c_{s}^{2} \rho+\frac{c_{s}^{2} G}{2} \psi^{2}
$$

In this equation, the right term is comprised of the ideal and non-ideal fluid behavior, respectively.

\section{Laplace test and surface tension estimation}

Among the fluid parameters, the surface tension is directly related to $G$ and the density profile at the interface of the two fluids, and they are expressed by the equation of state ${ }^{44}$, but it cannot be determined directly and requires simulations. Taking into account simulation with circle droplets in different radii, the surface tension is equal to the slope of the inside and outside pressure differences

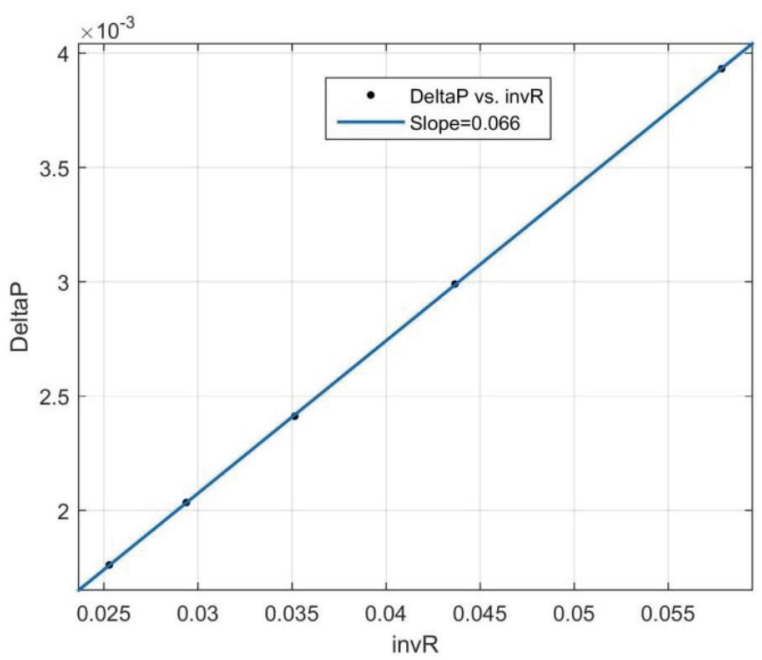

Fig. 1 - Laplace test and surface tension estimation

of the droplets and the inverse of the radiii ${ }^{45}$. The periodic boundary conditions are on the four sides of the model, and a simulation is started by considering a square droplet of the first fluid inside the second fluid. After reaching the equilibrium condition, the radius of the circle and inside and outside pressure are calculated. By repeating this simulation and calculating the pressure difference in different radii, the surface tension is obtained from the slope of the pressure difference versus inversion of the radius. To calculate the pressure, Equation (10) is used. To measure the radius graphically, the density graph of the two fluids is drawn from the center of the network toward the outside direction by radius of $R=\sqrt{(i-L / 2)^{2}+(j-L / 2)^{2}}$ and the intersection point is equal to the radius of the droplet. The following formulae is also used to calculate the radius of the droplet in which $\bar{\rho}$ is the average density in the entire network for the first fluid, or the fluid that forms the droplet ${ }^{46}$.

$$
\rho_{1 \max } \pi R^{2}+\left(L^{2}-\pi R^{2}\right) \rho_{1 \min }=\bar{\rho} L^{2}
$$

The variations in the surface tension are dependent on the $G$ and the density of the fluids. Smaller $G$ causes and bigger $G$ causes instability. Therefore, the size of $G$ must be large enough not only to avoid the thickening of the interface, but also to stabilize it enough. The results of this simulation for two fluids with a density and relaxation time of 1 and $G=$ 3 are shown in Fig. 1, and the estimated surface tension is 0.066 .

\section{Laplace test, Contact angle determination}

By substitution of surface tension and molecular adhesion of two fluids in the Yang equation (Equation (12)) with the parameters of LBM, the Equation (13) is obtained. 


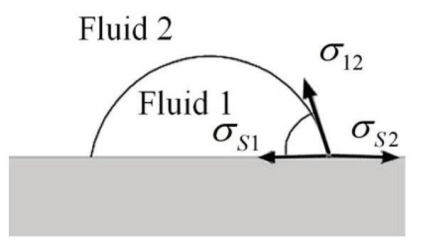

Fig. 2 - Contact angle

$$
\begin{aligned}
\cos \theta & =\frac{\sigma_{S 2}-\sigma_{S 1}}{\sigma_{12}} \\
\cos \theta_{1} & =\frac{G_{a d s 2}-G_{a d s 1}}{G_{c} \frac{\rho_{1}-\rho_{2}}{2}}
\end{aligned}
$$

In equation (13), $\rho_{1}$ and $\rho_{2}$ are the densities of the first fluid and the second dissolved fluid, respectively. For the values of $G_{a d s 1}$ and $G_{a d s 2}$, different intervals exist, but the least error in using this equation and LBM simulation occurs when the adhesion parameters of the two fluids are equal with opposite signs. The positive and negative signs indicate repulsion and attraction, respectively. The results of the contact angle determination with various $G$ are shown in Fig. 3. In this simulation, a rectangular droplet with the size of $4 \%$ of cross-sectional area is placed in $100 \times 200$ lattice network. The density of each fluid is 2 and the density of 0.06 is considered for dissolved fluid. The viscosity of both fluids is 0.1667 . The results of this simulation are in complete correlation with the results of Huang et al. ${ }^{47}$

\section{Results and discussion}

\section{Grid independence test}

The effect of grid size on the simulation has been considered with four sizes of porous media. The results of this simulation with Reynolds num- ber of 168 for fluids with surface tension of 0.666 , which resulted from the $G$ of 3, relaxation time of 1 , density of 1 for both fluids (they are equivalent to viscosity ratio of 1 ), and adhesion coefficient of 0 or 90 degrees contact angle (they are equivalent to natural wettability) are shown in Fig. 4. As it can be seen, in four grid sizes of $255 \times 255,561 \times 561$, $937 \times 937$, and $1403 \times 1403$, the fluid front only had a slightly different shape in grid size of $255 \times 255$. This deviation can also be seen in Fig. 4(e), in which the water saturation is plotted versus dimensionless time $\left(t_{D}=t / t_{\text {breakthrough }}\right)$, and there are no significant changes in other sizes. Therefore, these figures suggest that the grid sizes above $561 \times 561$ have negligible impact on water saturation and fluid front shape.

\section{Flow regimes}

The fluid front in porous media depends on a variety of factors. In this study, the viscous fingering is considered according to three parameters of the displacing fluid, including the discharge or capillary number $(\mathrm{Ca}=\rho v \mathrm{~V} / \sigma)$, dynamic viscosity ratio $(\mu=\rho v)$, and surface tension $(\sigma)$. Fig. 5 shows the viscous fingering in a microfracture, in which $L$ and $H$ are the fracture's length and width, respectively. $W_{\text {in }}$ is the internal width, $W_{\text {out }}$ is the outer width, $T$ in the length, and $S$ is the length of the contact line of the viscous fingering.

Various parameters affect the viscous fingering. Fig. 6 shows the distribution of the fluid front shape with respect to the changes in the viscosity ratio and the capillary number that is divided into three different regions ${ }^{48}$. These results were also obtained by Color-Gradient model and Free-Energy model of LBM simulation and experimentally later ${ }^{34,49,50}$.

In the stable displacement region, the main force is derived from the viscosity of the displacing fluid, and the capillary effect and the pressure drop

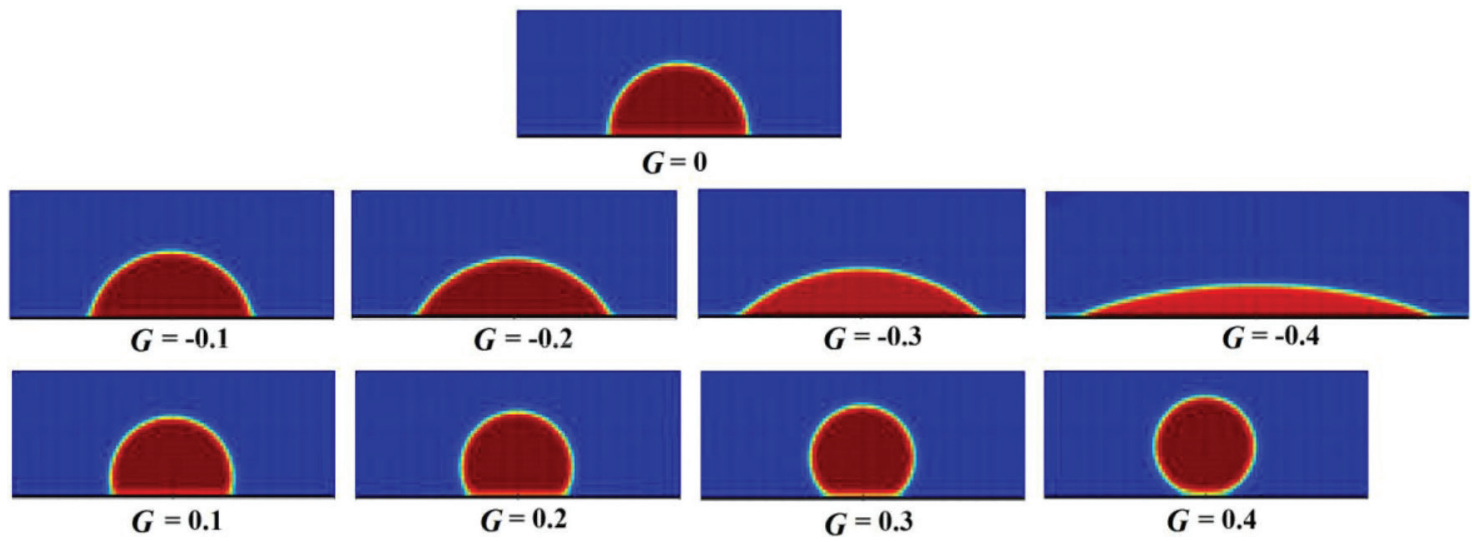

Fig. 3 - Simulation results of contact angle determination with different adhesion values 
a)

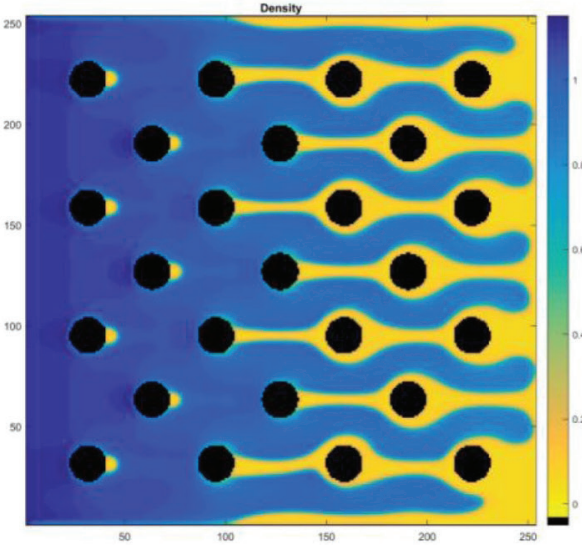

c)

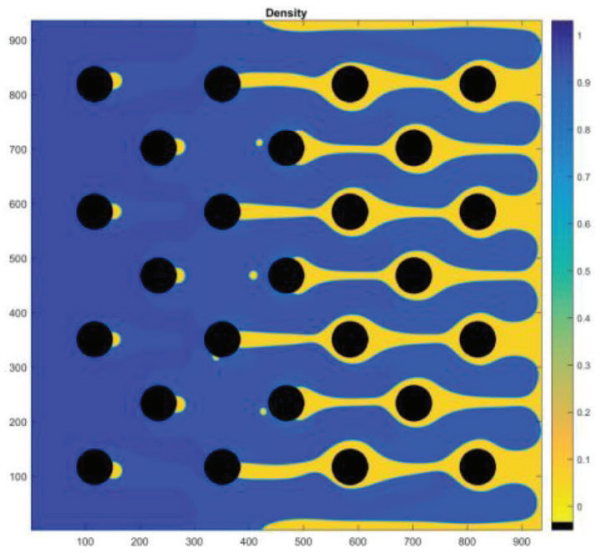

b)

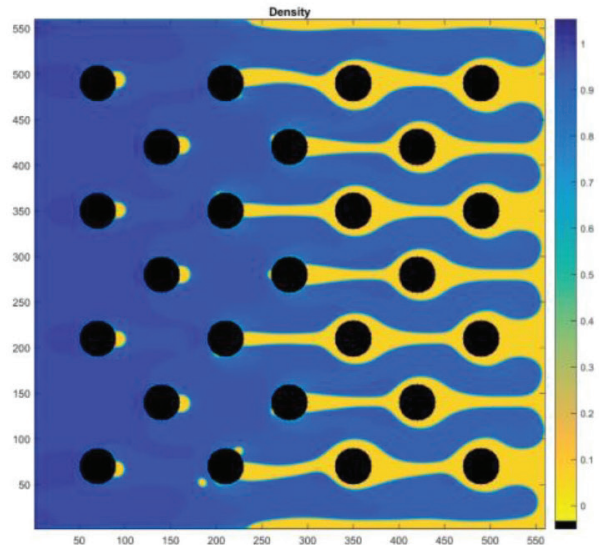

d)

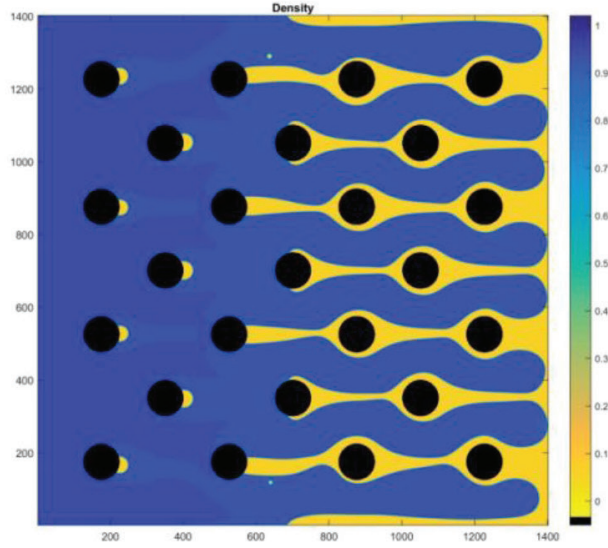

e)

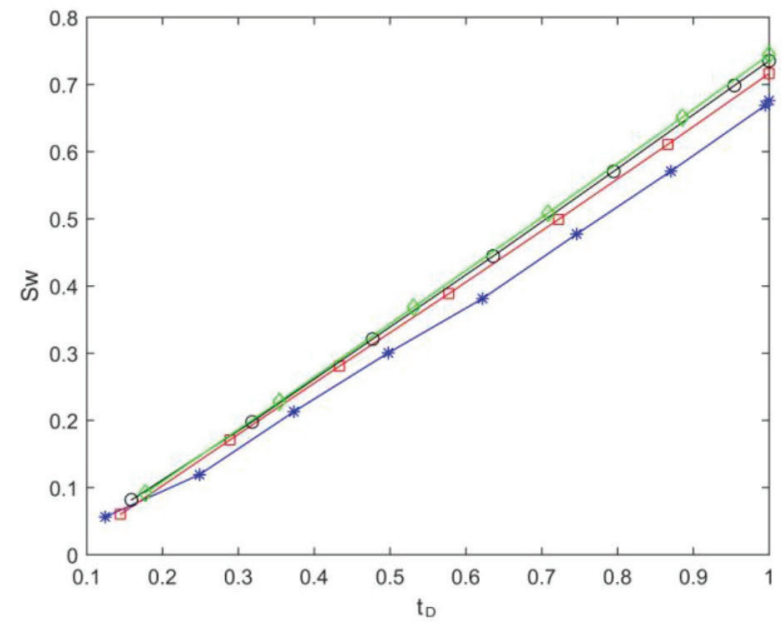

Fig. 4 - Grid size effects on fluid front profile at breakthrough time with grid size of (a) $255 \times 255$, (b) $561 \times 561$, (c) $937 \times 937$, (d) $1403 \times 1403$, and (e) comparison of water saturation for different dimensionless time for grid sizes of star: $255 \times 255$, square: $561 \times 561$, circle: $937 \times 937$, and diamond $1403 \times 1403$

are negligible. The shape of the fluid front is flat with few irregularities and fluid droplets are trapped in pore space ${ }^{48}$.

In the viscous fingering region, the main force is applied by the displaced fluid, and the capillary effect and the pressure drop are negligible. The viscous fingering is similar to tree branches without loops and extends to the entire porous media toward the outlet ${ }^{48}$.

In the capillary fingering region with low capillary numbers, viscosity is not noticeable for both fluids, and the main force is due to the capillary effect with a finger-like pattern, porous media extends in all directions, even to the opposite side and to- 


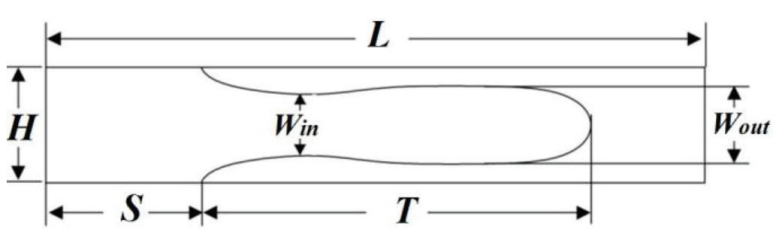

Fig. 5 - Fluid flow front and viscous fingering in a microfracture

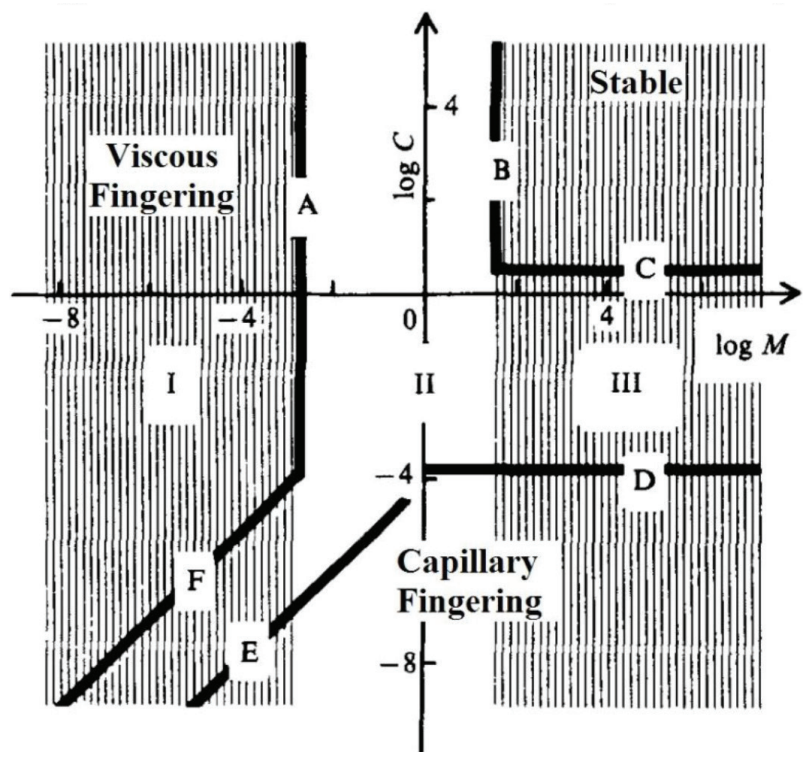

Fig. 6 - Fluid regimes and fluid front ${ }^{48}$

wards the entrance, which form loops of various sizes in which the displaced fluid is being trapped. The three boundaries among these three regions are dependent on pore size and porous media, but the overall shape does not change ${ }^{48}$.

Several studies have been conducted in this regard. According to the results, for a viscosity ratio more than one in low capillary numbers, the fluid is displaced by capillary fingering, in which the fluid tends to move towards the wider pores in different directions, and the fluid may move perpendicularly and even backward of the fluid flow direction. By the increase in capillary number, the displacement becomes stable. For a low viscosity ratio less than one in low capillary numbers, the capillary fingering is regenerated, while viscous fingering will prevail in high capillary numbers due to the injection of very low viscous fluid into the highly viscous fluid. In this case, there are several connected and disconnected flow paths toward the outlet in which the capillary force and the pressure drop in the fluid movement are negligible. The main force of the movement is the viscous force. In moderate capillary numbers, both the phenomena are observed and the region is known as Crossover Zone ${ }^{34,51,52}$. The simulation of current study takes place in the viscous fingering and in the transient zone of Fig. 6. The results of microfracture were consistent with
Hele-Shaw microchannel results ${ }^{26-30}$ and the same conditions were followed in the simulation of porous media.

\section{Viscous fingering and flow rate}

In immiscible displacement, the capillary force is dominant in the low discharge, leading to the formation of blobs trapping. By increasing capillary number and flow rate, the viscous effects are dominant and cause viscous fingering ${ }^{53,54}$ and it is effective in the displacement efficiency at the breakthrough time. According to the study of Liu et al., the increase in capillary number due to instability in the fingers of the fluid front, the injected fluid flow is not in a quasi-steady state and the slow decrease in displacement efficiency is observed, then, with the increase of the capillary number with quasi-steady state of flow, the displacement efficiency increases linearly ${ }^{32,49}$.

In this simulation, the Fracture width is 40 lattices, the Fracture length is 200 lattices, and the porosity of porous media is assigned to $90 \%$. A constant velocity boundary scheme proposed by Zou and He was used at both left inlet and right outlet of the model ${ }^{55}$ and the upper and lower and grains' solid wall was half-way bounce back boundary condition. The results of this simulation with velocities of $0.001,0.005,0.01,0.05,0.1$ for fluids with surface tension of 0.666 , which resulted from the $G$ of 3, relaxation time of 1 , density of 1 for both fluids, which are equivalent to viscosity ratio of 1 , and adhesion coefficient of 0 or 90 degrees contact angle, which are equivalent to natural wettability, are shown in Fig. 7. By applying very low velocities equivalent to low capillary numbers, the fluid front will have a slight curvature, and by increasing the flow rate, this curvature increases and breakthrough time decreases. As it can be seen, for better visualization of fluid front of the channel in higher flow rate, the contour lines of fluid front are plotted in 100 time steps instead of 500 time steps used in lower flow rate. Therefore, the increases in the velocity will persuade the fluid front from the piston form to the viscous fingering. However, as it can be seen, the amount of trapped droplets of the saturated fluid depends on the velocity, and most of them are observed at the velocity between the piston form front and the viscous fingering.

\section{Viscous fingering and viscosity ratio}

In this simulation, the Fracture width was 40 lattices, the Fracture length was 200 lattices, and the porosity of porous media was assigned to $90 \%$. A constant velocity boundary scheme proposed by Zou and $\mathrm{He}$ was used at both left inlet and right outlet of the model ${ }^{55}$ and the upper and lower and 
1)

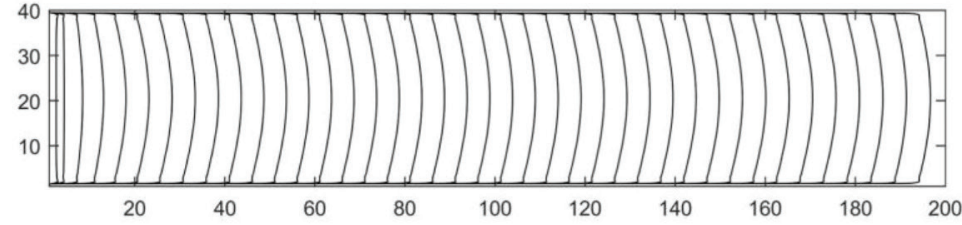

2)

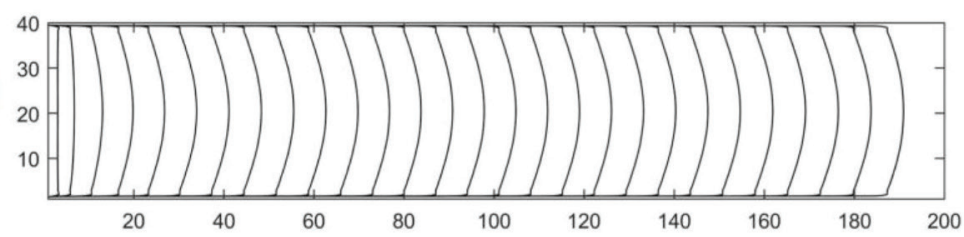

4)
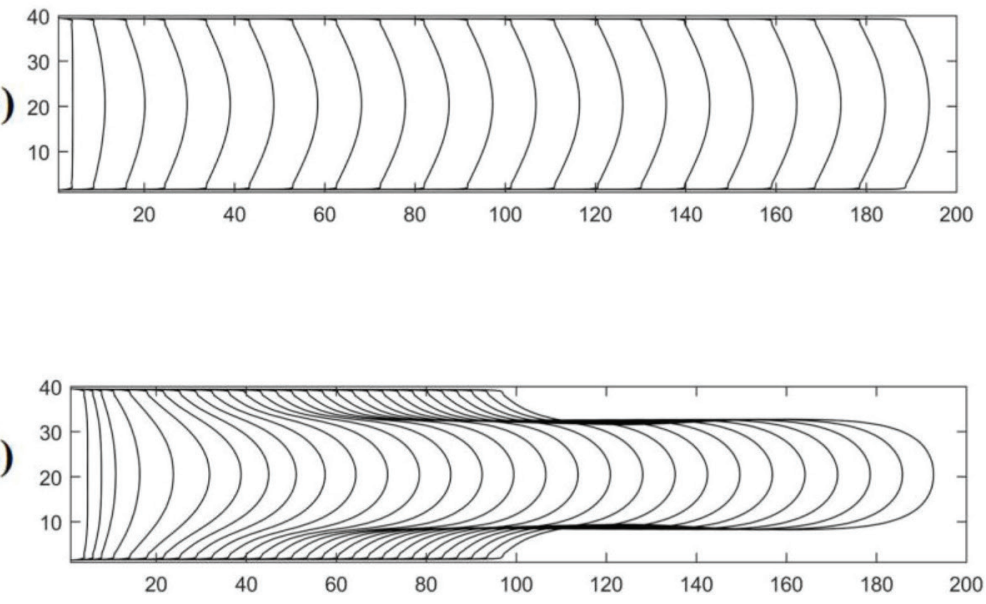

5)

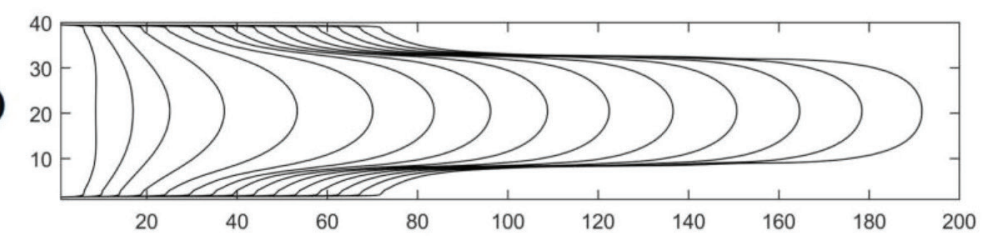

a
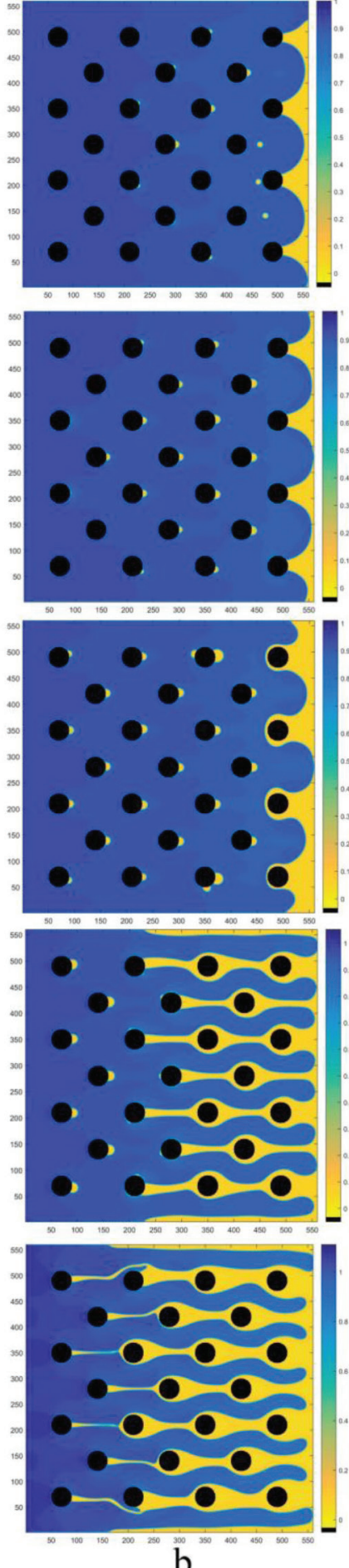

Fig. 7 - Flow rate effects on fluid front profile with the velocities of $u=0.001$ (1), $u=0.005$ (2), $u=0.01$ (3), $u=0.05$ (4), $u=0.1$ (5) in a microfracture (a) in the intervals of 500 time steps (1-3) and 100 time steps (4-5), and in porous media (b) in breakthrough time

grains' solid wall was half-way bounce back boundary condition. The density of both fluids was 1 , and the relaxation time of the displaced and displacing fluids were 1 and 0.666 , respectively. The viscosity ratio changed between $1 / 5$ and 1 , the capillary number was fixed at 0.063 , the wettability of the fluids was neutral (adhesion coefficient of 0 or 90 degrees contact angle), and $G$ was determined by the stability of the simulation. Due to the changes in $G$ and in the viscosity of the displacing fluid, the suitable velocity by fixing the capillary number was calculated and applied (Table 1). If the dynamic viscosity ratio, which is the ratio of the displacing fluid to the displaced fluid, is greater than 1 , the displacement 
Table 1 - Flow conditions for different viscosity ratios with capillary number of 0.063

\begin{tabular}{c|c|c|c|c|c}
\hline Viscosity ratio & Relaxation time of displacing fluid & Relaxation time of displaced fluid & $G$ & Surface tension & Velocity \\
\hline 1 & 1.000 & 1 & 3.0 & 0.066 & 0.025 \\
$1 / 2$ & 0.750 & 1 & 2.5 & 0.037 & 0.028 \\
$1 / 3$ & 0.666 & 1 & 2.0 & 0.019 & 0.021 \\
$1 / 4$ & 0.625 & 1 & 1.8 & 0.010 & 0.015 \\
$1 / 5$ & 0.600 & 1 & 1.7 & 0.010 & 0.018 \\
\hline
\end{tabular}

1)

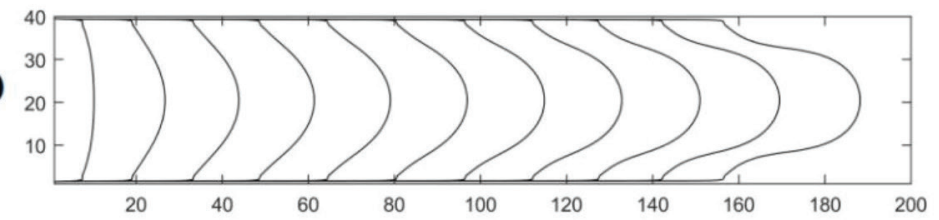

2)

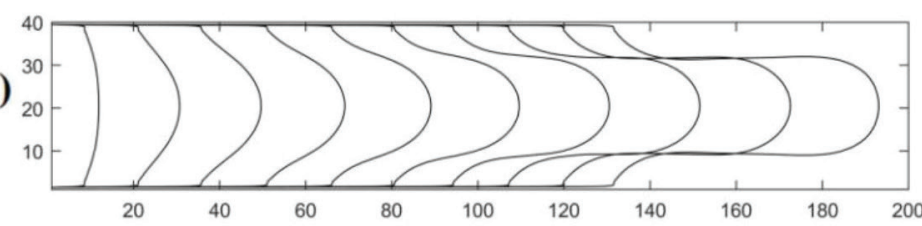

3)

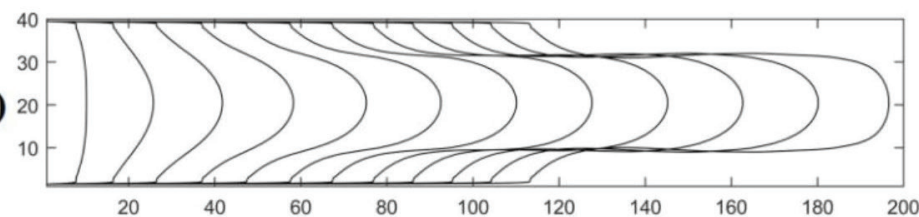

4)

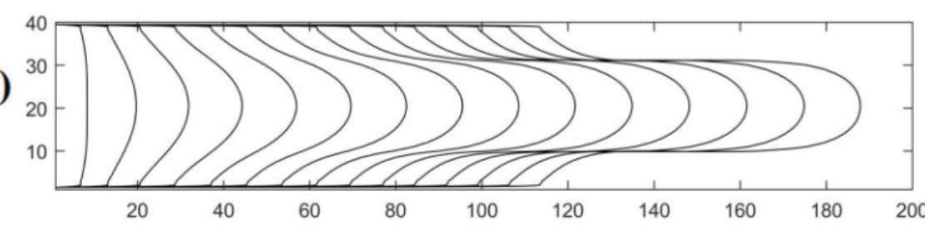

5)

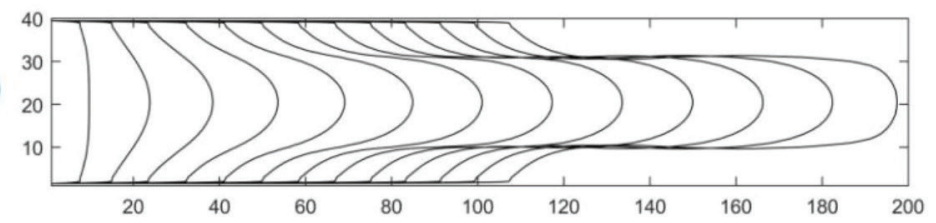

a
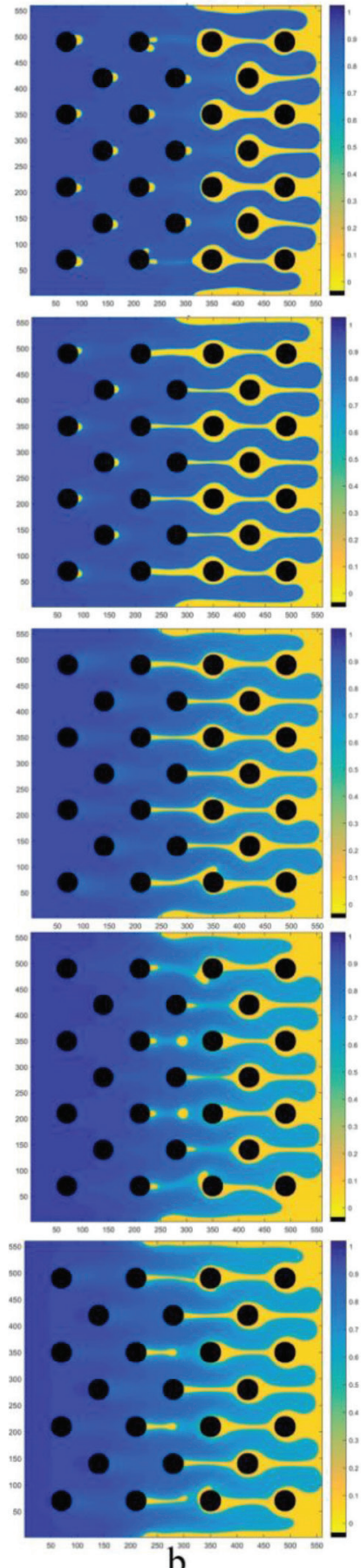

Fig. 8 - Effects of viscosity ratio on the fluid front profile with capillary number of 0.063 and the viscosity ratio of $M=1$ (1), $M=(1 / 2)(2), M=(1 / 3)(3), M=(1 / 4)(4), M=(1 / 5)$ (5) in a microfracture (a) in the intervals of 500 time steps and in porous media (b) in breakthrough time 
is called "favorable", and if it is less than 1 , the displacement is called "unfavorable" ". The results of this simulation by various viscosity ratios in the fracture and porous media are shown in Fig. 8. Viscous fingering length increases in both fracture and porous media. The breakthrough time decreases with the increase in the dynamic viscosity differences. It is important to note that Shan-Chen model of LBM is sensitive to the dynamic viscosity ratio, and it is unstable in viscosity ratios above 5. Fluid-fluid interaction coefficient $(G)$ lower than its critical value will yield a stable solution and higher values rather than this value rising until numerical instability occurs ${ }^{43}$. By higher density ratios, $G$ must be reduced to avoid instability and by Shan-Chen model of LBM (equation (7)), this ratio should be less than 5. This limitation has been partially removed using different equations of state ${ }^{56,57}$ and using different models of LBM, such as phase-field model $^{58}$.

According to the dynamic viscosity ratio, three zones are defined. In zone I, in which the dynamic viscosity ratio is very low, the viscous force of the displacing fluid in comparison to the viscous force of the displaced fluid is negligible. In zone II or the transitional zone in which the pressure drops, the viscosity of both fluids plays an important role. In zone III, dynamic viscosity ratio is high, the pressure drop due to the viscosity of displaced fluid is negligible and viscous force of the displacing fluid is dominant ${ }^{48}$. In a constant capillary number with low viscosity ratio and without gravity force, displacing a wetting fluid by a non-wetting fluid causes a thin viscous fingering pattern and occupies only small pore spaces. In some pore spaces, the displacing fluid is trapped, and is known as "daughter bubbles". By increasing the viscosity of displacing fluid or viscosity ratio, the thickness of the fingers and the daughter bubbles' size increase. With continuous increase, all pores will fill and the displacing fluid will not be trapped in the pores, and therefore, the capillary fingering or stable conditions will form ${ }^{49}$. According to Fig. 6, the simulation of this study takes place in the viscous fingering and in the transient zone. As it can be seen, with the decrease in the dynamic viscosity ratio, the viscous fingering becomes very thin and takes up only small pore spaces, while the trapped bubbles of displaced fluid decrease.

\section{Viscous fingering and wettability}

Considering the adhesion force between the fluid and the solid surface, its effects on the fluid front are important. Fluid injection, by assuming that the fracture and porous media are saturated with the second fluid, with different wettability equivalent to contact angles of $30,60,90,120,150$ degrees, is shown in Fig. 9. In this simulation, with the dynamic viscosity ratio of $(1 / 3)$, the density of both fluids was 1 , and the relaxation time of the displaced and displacing fluids were 1 and 0.666 , respectively. The fluid-fluid interaction coefficient $(G)$ was 2 , the width of the fracture was 40 lattices, the length of the fracture was 200 lattices, the porosity of porous media was $90 \%$, and the injection velocity was 0.021 . A constant velocity boundary scheme proposed by Zou and He was used at both left inlet and right outlet of the model ${ }^{55}$ and the upper and lower and grains' solid wall was half-way bounce back boundary condition. As it can be seen, contact angle or wettability was another factor affecting the shape of the fluid front due to its effects on the capillary force and the non-tendency of nonwet fluids to enter the pore spaces. By injecting fluid with neutral wettability, smaller bubbles of displaced fluid were trapped in the displacing fluid, and therefore, the dendritic structures had decreased. By increasing the wettability and decreasing the breakthrough time, these bubbles were absent and the flow regime became stable in piston form. The results are consistent with the results of Cottin and Liu et al. ${ }^{49,51}$ Liu et al. showed that in gas injection by the viscous fingering mechanism at the breakthrough time with different wettability, the sweep efficiency of the gas was a function of the wettability. By increasing the wettability from non-wet to neutral, the sweep efficiency decreased, and from a neutral fluid to wet, the sweep efficiency increased. In addition, the lowest sweep efficiency occurred with neutral wettability. The sweep efficiency obtained from this simulation (Fig. 10) confirmed this, and was in accordance with the results of Dong et $a l .32,49$

\section{Water flooding in a fracture and porous media saturated with Arvandan crude oil}

In this simulation, the width of fracture was 40 lattices, the length of the fracture was 200 lattices, porosity of porous media was $90 \%$, and the distilled water as the displacing fluid was 1 , and the density and the dynamic viscosity of Arvandan crude oil as the displaced fluid were 0.875 and 6 , respectively. The contact angle of water droplet on glass sheet in the oil-saturated media as the second fluid was $55^{\circ}$. A constant velocity boundary scheme proposed by Zou and He was used at both left inlet and right outlet of the model ${ }^{55}$ and the upper and lower and grains' solid wall was half-way bounce back boundary condition. After converting these parameters from SI units to the lattice units, the simulation was performed with a capillary number of 0.063. The results (Fig. 11) showed that the fluid front pattern is fingering and some bubbles of saturated fluid remain in the pores. 


\section{Conclusions}

The fluid front in EOR process of oil resources is important. Along with the experimental results, the use of accurate simulations, which are consistent with experimental results, will help petroleum engineers to assess petroleum reservoirs. Shan-
-Chen model of LBM is one of the mesoscopic-scale simulation tools by which we can study the fluid front in the pore scale.

In this study, the parameters of velocity, dynamic viscosity, with which the capillary number is defined, and wettability were investigated in formation of viscous fingering in a microfracture and a

1)

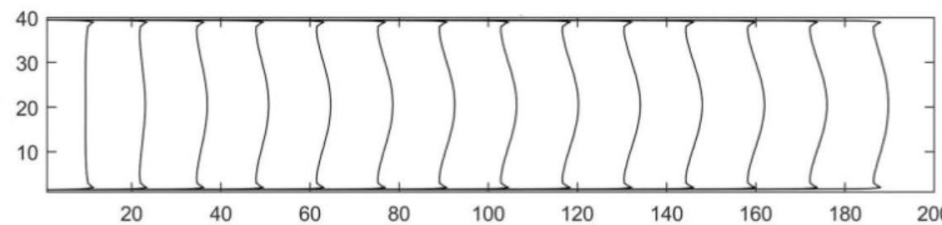

2)

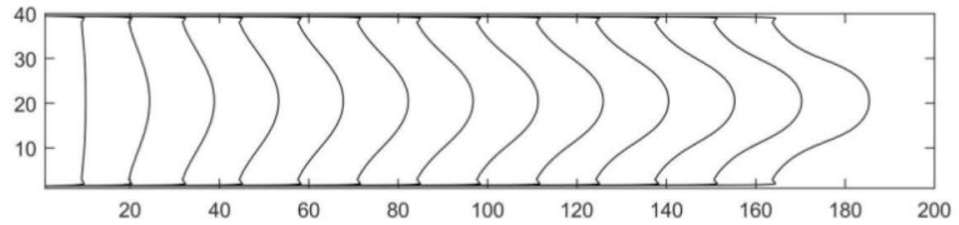

3)

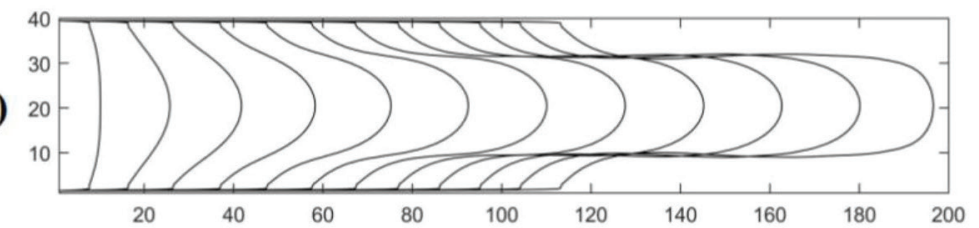

4)

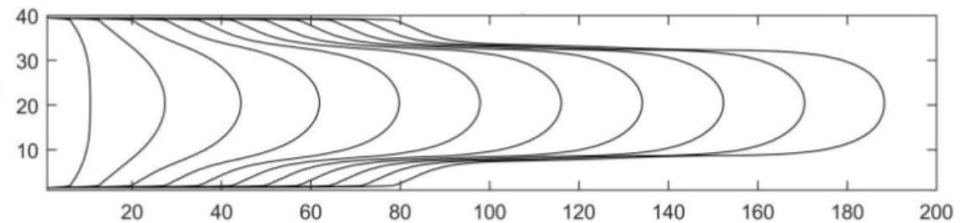

5)

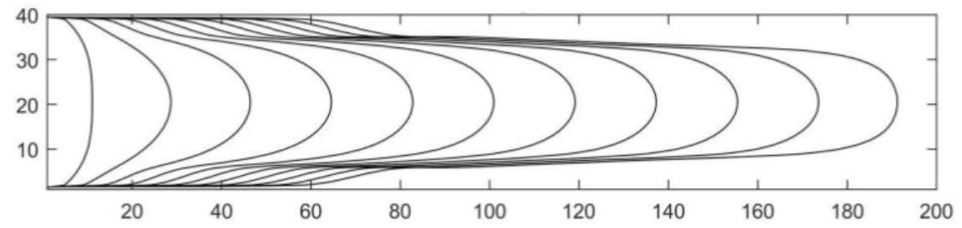

a

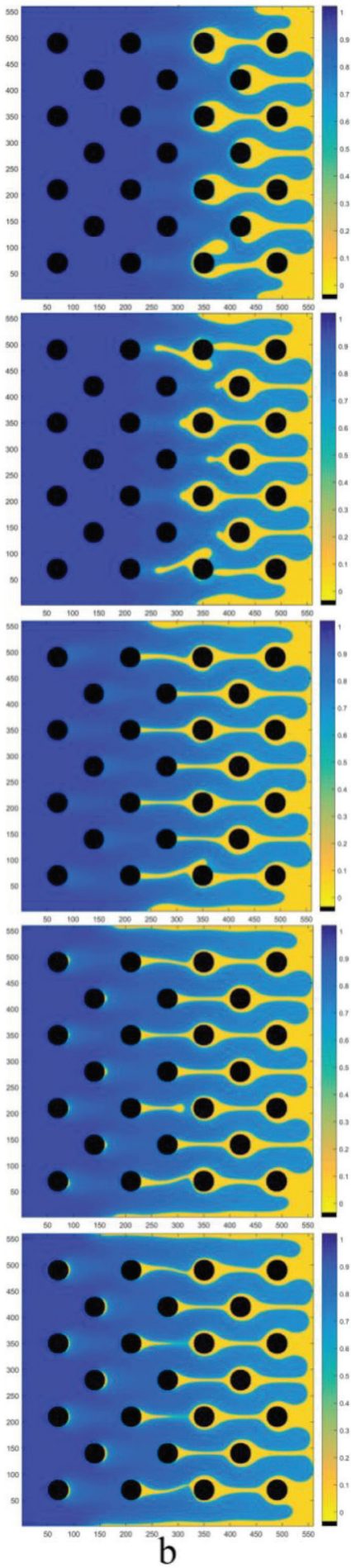

Fig. 9 - Wettability effects on fluid front profile with injection velocity of 0.021 , viscosity ratio of $1 / 3, G$ of 2 and the contact angles of $C A=30(1), C A=60(2), C A=90(3), C A=120(4), C A=150$ (5) degrees in a microfracture (a) in the intervals of 500 time steps and in porous media (b) in breakthrough time 


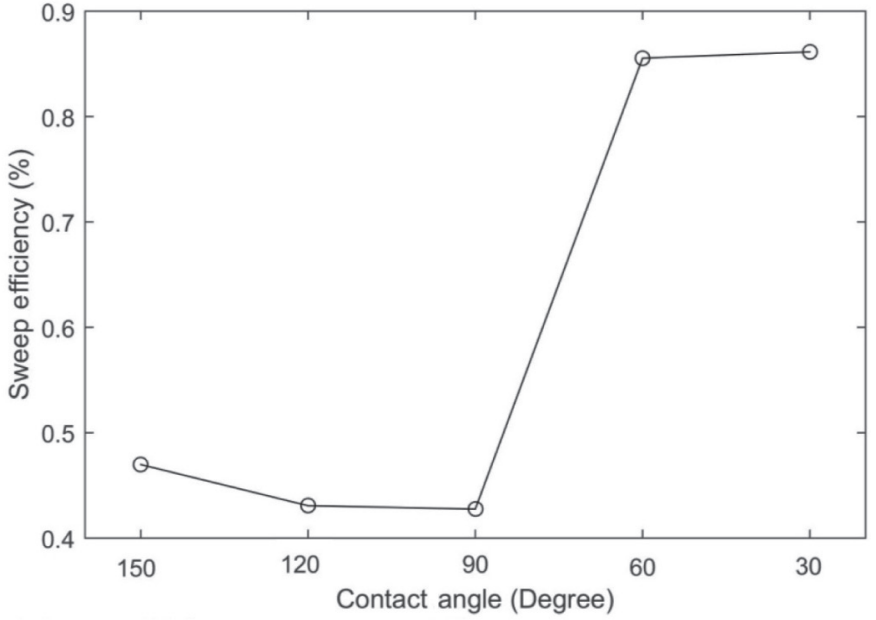

Fig. 10 - Sweep efficiency versus wettability

simple porous media. Independently, the increase in velocity and decrease in dynamic viscosity ratio resulted in the formation of a fingering pattern, while wettability reduced it. In addition, the least amount of sweep efficiency occured when the displacing fluid had neutral wettability.

The results of this simulation show the strength and accuracy of the Shan-Chen Model of LBM in simulating the multi-component immiscible displacement, EOR, and fluid front tracking in the pores of porous media.

\section{ACKNOWLEDGMENTS}

This article was extracted from a PhD thesis written by Yousef Shiri. Thanks to the faculty of petroleum engineering of Amirkabir University of Technology, the Faculty of Petroleum Engineering and Mechanical Engineering of Shahrood University of Technology and the Research and Development branch of Petroleum Ministry for the provision of educational and financial services. We are grateful to Dr. Esmail Shakeri and Dr. Mohammad Mohammadi Fard for their priceless guidance.

\section{References}

1. Moore, M. G., Fluctuations and pinch-offs observed in viscous fingering, AIP Conf. Proc. 676 (2003) 189. doi: https://doi.org/10.1063/1.1612212

2. Ledesma-Aguilar, R., Quevedo-Reyes, M., Poiré, E. C., Hernández-Machado, A. Lateral instability in normal viscous fingers, Phys. Rev. E - Stat. Nonlinear, Soft Matter Phys. 71 (2005) 016312.

doi: https://doi.org/10.1103/PhysRevE.71.016312

3. Torralba, M., Ortín, J., Hernández-Machado, A., Poiré, E. $C$., Fluctuations in Saffman-Taylor fingers with quenched disorder, Phys. Rev. E - Stat. Nonlinear, Soft Matter. Phys. 73 (2006) 046302.

doi: https://doi.org/10.1103/PhysRevE.73.046302

4. Quevedo-Reyes, M., Hernández-Machado, A., Poiré, E. C. Phase field approach to spatial perturbations in normal Saffman-Taylor fingers, Phys. Rev. E - Stat. Nonlinear, Soft Matter. Phys. 73 (2006) 066308. doi: https://doi.org/10.1103/PhysRevE.73.066308

5. Stokes, J. P., Weitz, D. A., Gollub, J. P., Dougherty, A., Robbins, M. O., Chaikin, P. M., Lindsay, H. M., Interfacial satbility of immiscible displacement in a porous medium. Phys. Rev. Lett. 57 (1986) 1718. doi: https://doi.org/10.1103/PhysRevLett.57.1718

6. Homsy, G. M., Viscous fingering in porous media, Annu. Rev. Fluid Mech. 19 (1987) 271. doi: https://doi.org/10.1146/annurev.fl.19.010187.001415

7. Cinar, Y., Jessen, K., Berenblyum, R., Juanes, R., Orr, F. M. $J$., An experimental and numerical investigation of crossflow effects in two-phase displacements, Spe 11 (2006) 216. doi: https://doi.org/10.2118/90568-PA

8. Maaref, S., Rokhforouz, M. R., Ayatollahi, S., Numerical investigation of two phase flow in micromodel porous media: Effects of wettability, heterogeneity, and viscosity, Can. J. Chem. Eng. 95 (2017) 1213. doi: https://doi.org/10.1002/cjce.22762

9. Kärger, J., Flow and transport in porous media and fractured rock, Zeitschrift für Physikalische Chemie 194 (1996) 135.

doi: https://doi.org/10.1524/zpch.1996.194.Part_1.135a

10. Nazari, Nazari, M., Kayhani, M., A comparative solution of natural convection in an open cavity using different boundary conditions via the Lattice Boltzmann method, J. Heat Mass 3 (2016) 115. doi: 10.22075/JHMTR.2016.363

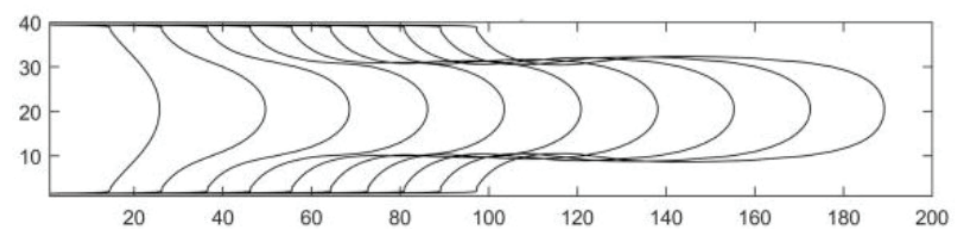

a

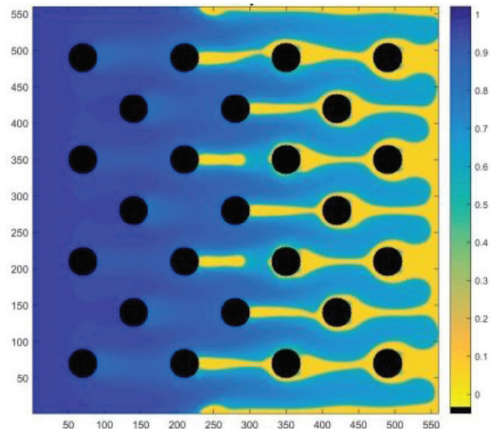

b

Fig. 11 - Water flooding and viscous fingering in a microfracture in the intervals of 500 time steps and in a simple porous media (b) in breakthrough time, both saturated with Arvandan crude oil 
11. Delouei, A. A., Nazari, M., Kayhani, M. H., Ahmadi, G., A non-Newtonian direct numerical study for stationary and moving objects with various shapes: An immersed boundary - Lattice Boltzmann approach, J. Aerosol Sci. 93 (2016) 45 . doi: https://doi.org/10.1016/j.jaerosci.2015.11.006

12. Delouei, a. A., Nazari, M., Kayhani, M. H., Succi, S. Immersed boundary - thermal Lattice Boltzmann methods for non-Newtonian flows over a heated cylinder: A comparative study, Commun. Comput. Phys. 18 (2015) 489. doi: https://doi.org/10.4208/cicp.060414.220115a

13. Sedaghat, M. H., Shahmardan, M. M., Norouzi, M., Jayathilake, P. G., Nazari, M., Numerical simulation of muco-ciliary clearance: Immersed boundary-lattice Boltzmann method, Comput. Fluids 131 (2016) 91. doi: https://doi.org/10.1016/j.compfluid.2016.03.015

14. Chen, S., Doolen, G. D., Lattice Boltzmann Method for fluid flows, Annu. Rev. Fluid Mech. 30 (2003) 329. doi: https://doi.org/10.1146/annurev.fluid.30.1.329

15. Nabovati, A., Pore level simulation of single and two phase flow in porous media using Lattice Boltzmann method. Doctoral dissertation, University of New Brunswick, Department of Mechanical Engineering, 2009.

16. Gunstensen, A. K., Rothman, D. H., Zaleski, S., Zanetti, G., Lattice Boltzmann model of immiscible fluids, Phys. Rev. A 43 (1991) 4320. doi: https://doi.org/10.1103/PhysRevA.43.4320

17. Shan, $X$., Chen, $H$., Lattice Boltzmann model for simulating flows with multiple phases and components, Phys. Rev. E 47 (1993) 1815. doi: https://doi.org/10.1103/PhysRevE.47.1815

18. Gonnella, G., Lamura, A., Sofonea, V., Lattice Boltzmann simulation of thermal nonideal fluids, Phys. Rev. E Stat. Nonlin. Soft Matter. Phys. 76 (2007) 36703. doi: https://doi.org/10.1103/PhysRevE.76.036703

19. He, X., Shan, X., Doolen, G. D., Discrete Boltzmann equation model for nonideal gases, Phys. Rev. E 57 (1998) R13. doi: https://doi.org/10.1103/PhysRevE.57.R13

20. Ahrenholz, B., Tölke, J., Lehmann, P., Peters, A., Kaestner, $A$., Krafczyk, M., Durner, W., Prediction of capillary hysteresis in a porous material using Lattice-Boltzmann methods and comparison to experimental data and a morphological pore network model, Adv. Water Resour. 31 (2008) 1151. doi: https://doi.org/10.1016/j.advwatres.2008.03.009

21. Sbragaglia, M., Benzi, R., Biferale, L., Succi, S., Sugiyama, $K$., Toschi, F., Generalized Lattice Boltzmann method with multirange pseudopotential, Phys. Rev. E - Stat. Nonlinear, Soft Matter. Phys. 75 (2007) 26702. doi: https://doi.org/10.1103/PhysRevE.75.026702

22. Guo, Z., Shu, C., Lattice Boltzmann method and its applications in engineering, Vol. 3, World Scientific Publishing, 2013. doi: https://doi.org/10.1007/s11434-009-0681-6

23. Saffman, P. G., Taylor, G., The Penetration of a fluid into a porous medium or hele-shaw cell containing a more viscous liquid, Proc. R. Soc. London Ser. A, Math. Phys. Sci. 245 (1958) 312. doi: https://doi.org/10.1098/rspa.1958.0085

24. Foroughi, H., Abbasi, A., Das, K. S., Kawaji, M., Immiscible displacement of oil by water in a microchannel: Asymmetric flow behavior and nonlinear stability analysis of core-annular flow, Phys. Rev. E - Stat. Nonlinear, Soft Matter Phys. 85 (2012) 026309. doi: https://doi.org/10.1103/PhysRevE.85.026309
25. Ramachandran, R., Stability and onset of two-dimensional viscous fingering in immiscible fluids. arXiv preprint arXiv: $1704.02674,2017$ doi: https://arxiv.org/abs/1704.02674

26. Kang, Q., Zhang, D., Chen, S., Immiscible displacement in a channel: Simulations of fingering in two dimensions, Adv. Water Resour. 27 (2004) 13. doi: https://doi.org/10.1016/j.advwatres.2003.10.002

27. Dong, B., Yan, Y. Y., Li, W., Song, Y., Lattice Boltzmann simulation of viscous fingering phenomenon of immiscible fluids displacement in a channel, Comput. Fluids 39 (2010) 768.

doi: https://doi.org/10.1016/j.compfluid.2009.12.005

28. Yang, J., Boek, E. S., A comparison study of multi-component Lattice Boltzmann models for flow in porous media applications, Comput. Math. with Appl. 65 (2013) 882. doi: https://doi.org/10.1016/j.camwa.2012.11.022

29. Shi, Y., Tang, G. H., Simulation of Newtonian and non-Newtonian rheology behavior of viscous fingering in channels by the Lattice Boltzmann method, Comput. Math. with Appl. 68 (2014) 1279. doi: https://doi.org/10.1016/j.camwa.2014.08.024

30. Swain, P. A. P., Karapetsas, G., Matar, O. K., Sahu, K. C., Numerical simulation of pressure-driven displacement of a viscoplastic material by a Newtonian fluid using the Lattice Boltzmann method, Eur. J. Mech. B/Fluids 49 (2015) 197. doi: https://doi.org/10.1016/j.euromechflu.2014.08.010

31. Mahabadian, M. A., Ghayyem, M. A., Jamialahmadi, M., Multicomponent multiphase Lattice-Boltzmann modeling of fingering during immiscible displacement, Energy Sources, Part A Recover. Util. Environ. Eff. 37 (2015) 642. doi: https://doi.org/10.1080/15567036.2011.585377

32. Dong, B., Yan, Y. Y., Li, W. Z., LBM simulation of viscous fingering phenomenon in immiscible displacement of two fluids in porous media, Transp. Porous Media 88 (2011) 293. doi: https://doi.org/10.1007/s11242-011-9740-y

33. Ghassemi, A., Pak, A., Numerical study of factors influencing relative permeabilities of two immiscible fluids flowing through porous media using Lattice Boltzmann method, J. Pet. Sci. Eng. 77 (2011) 135. doi: https://doi.org/10.1016/j.petrol.2011.02.007

34. Zhang, C., Oostrom, M., Wietsma, T. W., Grate, J. W., Warner, $M$. G., Influence of viscous and capillary forces on immiscible fluid displacement: Pore-scale experimental study in a water-wet micromodel demonstrating viscous and capillary fingering, Energy and Fuels 25 (2011) 3493. doi: https://doi.org/10.1021/ef101732k

35. Huang, H., Wang, L., Lu, X. Y., Evaluation of three Lattice Boltzmann models for multiphase flows in porous media, Comput. Math. with Appl. 61 (2011) 3606. doi: https://doi.org/10.1016/j.camwa.2010.06.034

36. Yan, $Y . Y$., Zu, Y. Q., Dong, B., LBM, a useful tool for mesoscale modelling of single-phase and multiphase flow, Appl. Therm. Eng. 31 (2011) 649. doi: https://doi.org/10.1016/j.applthermaleng.2010.10.010

37. Chen, L., Kang, Q., Mu, Y., He, Y. L., Tao, W. Q., A critical review of the pseudopotential multiphase Lattice Boltzmann model: Methods and applications, Int. J. Heat Mass Transf. 76 (2014) 210. doi: https://doi.org/10.1016/j.ijheatmasstransfer.2014.04.032

38. Liu, H., Zhang, Y., Valocchi, A. J., Lattice Boltzmann simulation of immiscible fluid displacement in porous media: Homogeneous versus heterogeneous pore network, Phys. Fluids 27 (2015) 052103. doi: https://doi.org/10.1063/1.4921611 
39. Broadwell, J. E., Shock structure in a simple discrete velocity gas, Phys. Fluids 7 (1964) 1243. doi: https://doi.org/10.1063/1.1711368

40. McNamara, G. R., Zanetti, G., Use of the Boltzmann equation to simulate lattice-gas automata, Phys. Rev. Lett. 61 (1988) 2332. doi: https://doi.org/10.1103/PhysRevLett.61.2332

41. Bahr, D. B., Rundle, J. B., Theory of Lattice Boltzmann simulations of glacier flow, Journal of Glaciology 41 (1995) 634. doi: https://doi.org/10.3189/S0022143000034948

42. He, X., Luo, L., Theory of the Lattice Boltzmann method: From the Boltzmann equation to the lattice Boltzmann equation, Phys. Rev. E 56 (1997) 6811. doi: https://doi.org/10.1103/PhysRevE.56.6811

43. Huang, H., Sukop, M. C., Lu, X.-Y., Multiphase Lattice Boltzmann methods: Theory and application. John Wiley \& Sons, 2015 doi: https://doi.org/10.1002/9781118971451

44. Zhang, R., He, X., Chen, S., Interface and surface tension in incompressible Lattice Boltzmann multiphase model, Comput. Phys. Commun. 129 (2000) 121. doi: https://doi.org/10.1016/S0010-4655(00)00099-0

45. Rahmani, A., Ashrafizaade, M., Rahmati, A., Meysam, S. Evaluation of Shan-Chen Lattice Boltzmann model ability on simulation of multiphase and multicomponent flows, Conf. Semnan. Ac. Ir (2014) 19.

46. Hou, S. L., Shan, X. W., Zou, Q. S., Doolen, G. D., Soll, W. $E$., Evaluation of two Lattice Boltzmann models for multiphase flows, J. Comput. Phys. 138 (1997) 695. doi: https://doi.org/10.1006/jcph.1997.5839

47. Huang, H., Thorne, D. T., Schaap, M. G., Sukop, M. C. Proposed approximation for contact angles in Shan-andChen-type multicomponent multiphase Lattice Boltzmann models, Phys. Rev. E - Stat. Nonlinear, Soft Matter Phys. 76 (2007) 66701. doi: https://doi.org/10.1103/PhysRevE.76.066701

48. Lenormand, R., Touboul, E., Zarcone, C., Numerical models and experiments on immiscible displacements in porous media, J. Fluid Mech. 189 (1988) 165. doi: https://doi.org/10.1017/S0022112088000953

49. Liu, H., Valocchi, A. J., Kang, Q., Werth, C., Pore-scale simulations of gas displacing liquid in a homogeneous pore network using the Lattice Boltzmann method, Transp. Porous Media 99 (2013) 555 doi: https://doi.org/10.1007/s11242-013-0200-8
50. Huang, H., Huang, J. J., Lu, X. Y., Study of immiscible displacements in porous media using a color-gradient-based multiphase Lattice Boltzmann method, Comput. Fluids 93 (2014) 164 . doi: https://doi.org/10.1016/j.compfluid.2014.01.025

51. Cottin, C., Bodiguel, H., Colin, A., Drainage in two-dimensional porous media: From capillary fingering to viscous flow, Phys. Rev. E - Stat. Nonlinear, Soft Matter Phys. 82 (2010) 046315. doi: https://doi.org/10.1103/PhysRevE.82.046315

52. Wang, Y., Zhang, C., Wei, N., Oostrom, M., Wietsma, T. W., $\mathrm{Li}, \mathrm{X}$., Bonneville, A., Experimental study of crossover from capillary to viscous fingering for supercritical $\mathrm{CO}_{2}$-water displacement in a homogeneous pore network, Environ. Sci. Technol. 47 (2013) 212. doi: https://doi.org/10.1021/es3014503

53. Silva, M., Dawe, R., Effects of permeability and wettability heterogeneities on flow in porous media, SPE Latin American and Caribbean Petroleum Engineering Conference, 27-30 April (2003). doi: https://doi.org/10.2118/81164-MS

54. Holtzman, R., Effects of pore-scale disorder on fluid displacement in partially-wettable porous media, Nat. Publ. Gr. 6 (2016) 36221 doi: https://doi.org/10.1038/srep36221

55. Zou, Q., He, $X$., On pressure and velocity boundary conditions for the Lattice Boltzmann BGK model, Phys. Fluids 9 (1997) 1591 doi: https://doi.org/10.1063/1.869307

56. Yuan, P., Schaefer, L., Equations of state in a Lattice Boltzmann model, Phys. Fluids 18 (2006) 1. doi: https://doi.org/10.1063/1.2187070

57. Abadi, H., Ghaderi, A., Nazari, M., Simulation of fluid penetration with high density ratio in layered porous media with Lattice Boltzmann model by using equations of state, Mech. Eng. 48 (2016) 55. doi: http://dx.doi.org/10.22060/mej.2016.596

58. Li, Q., Luo, K. H., Kang, Q. J., He, Y. L., Chen, Q., Liu, Q., Lattice Boltzmann methods for multiphase flow and phasechange heat transfer, Prog. Energy Combust. Sci. 52 (2016) 62 . doi: https://doi.org/10.1016/j.pecs.2015.10.001 